\title{
Relationship Between Literacy, Knowledge, Self-Care Behaviors, and Heart Failure-Related Quality of Life Among Patients With Heart Failure
}

\author{
Aurelia Macabasco-O'Connell, PhD, RN, ACNP', Darren A. DeWalt, MD, MPH ${ }^{2,3}$, \\ Kimberly A. Broucksou, MSW, MPA' ${ }^{2}$, Victoria Hawk, MPH, RD, LDN², David W. Baker, MD, MPH', \\ Dean Schillinger, $M D^{6}$, Bernice Ruo, MD, MAS ${ }^{5}$, Kirsten Bibbins-Domingo, $P h D, M D^{6}$, \\ George M. Holmes, $P h D^{2,4}$, Brian Erman, BA, MS ${ }^{2}$, Morris Weinberger, $P h D^{4}$, \\ and Michael Pignone, MD, $\mathrm{MPH}^{2,3}$
}

'UCLA School of Nursing, University of California, Olive View-UCLA Medical Center Los Angeles, Los Angeles, CA, USA; ${ }^{2}$ Cecil G. Sheps Center for Health Services Research, University of North Carolina at Chapel Hill, Chapel Hill, NC, USA; ${ }^{3}$ Division of General Internal Medicine, University of North Carolina at Chapel Hill, Chapel Hill, NC, USA; ${ }^{4}$ Health Policy and Management, University of North Carolina at Chapel Hill, Chapel Hill, NC, USA; ${ }^{5}$ Feinberg School of Medicine, Northwestern University, Chicago, II, USA; ${ }^{6}$ Division of General Internal Medicine and Center for Vulnerable Populations, Department of Medicine at San Francisco General Hospital, University of California San Francisco, San Francisco, CA, USA.

BACKGROUND: We sought to examine the relationship between literacy and heart failure-related quality of life (HFGOL), and to explore whether literacy-related differences in knowledge, self-efficacy and/or self-care behavior explained the relationship.

METHODS: We recruited patients with symptomatic heart failure (HF) from four academic medical centers. Patients completed the short version of the Test of Functional Health Literacy in Adults (TOFHLA) and questions on HF-related knowledge, HF-related selfefficacy, and self-care behaviors. We assessed HFGOL with the Heart Failure Symptom Scale (HFSS) (range 0100), with higher scores denoting better quality of life. We used bivariate (t-tests and chi-square) and multivariate linear regression analyses to estimate the associations between literacy and HF knowledge, self-efficacy, self-care behaviors, and HFQOL, controlling for demographic characteristics. Structural equation modeling was conducted to assess whether general HF knowledge, salt knowledge, self-care behaviors, and self-efficacy mediated the relationship between literacy and HFQOL.

RESULTS: We enrolled 605 patients with mean age of 60.7 years; $52 \%$ were male; $38 \%$ were African-American and $16 \%$ Latino; $26 \%$ had less than a high school education; and $67 \%$ had annual incomes under $\$ 25,000$. Overall, $37 \%$ had low literacy (marginal or inadequate on TOFHLA). Patients with adequate literacy had higher general HF knowledge than those with low literacy (mean 6.6 vs. 5.5, adjusted difference 0.63 , p< 0.01 ), higher self-efficacy (5.0 vs. 4.1 , adjusted difference $0.99, \mathrm{p}<0.01$ ), and higher prevalence of key self-care behaviors $(p<0.001)$. Those with adequate literacy had better HFQOL scores compared to those with low literacy (63.9 vs. 55.4, adjusted difference $7.20, \mathrm{p}<0.01$ ), but

Received July 9, 2010

Revised November 22, 2010

Accepted February 10, 2011

Published online March 3, 2011 differences in knowledge, self-efficacy, and self-care did not mediate this difference in HFQOL.

CONCLUSION: Low literacy was associated with worse HFQOL and lower HF-related knowledge, self-efficacy, and self-care behaviors, but differences in knowledge, self-efficacy and self-care did not explain the relationship between low literacy and worse HFGOL.

KEY WORDS: literacy; self-care; quality of life; heart failure.

J Gen Intern Med 26(9):979-86

DOI: $10.1007 / \mathrm{s} 11606-011-1668-\mathrm{y}$

(C) The Author(s) 2011. This article is published with open access at Springerlink.com

\section{BACKGROUND}

Heart failure (HF) is a common chronic illness associated with substantial morbidity and mortality, poor quality of life (QOL), and frequent hospitalizations ${ }^{1}$. Multiple barriers, including communication difficulties, lack of self-management support, poverty, lack of health insurance, and poor access to appropriate health care can contribute to worse HF outcomes.

Low literacy may be an important cause of adverse health outcomes for many chronic conditions, including HF. Low literacy is common; according to the 2003 National Assessment of Adult Literacy, 35\% of the U.S. population has belowbasic or basic health-related literacy ${ }^{2}$. Studies have shown an association between limited literacy and poorer knowledge about health conditions ${ }^{3,4}$, less use of preventive services ${ }^{5}$, higher hospitalization rates $^{6,7}$, increased mortality ${ }^{8,9}$, and poorer self-reported health status ${ }^{6,10,11}$.

Although literacy is associated with many adverse health outcomes, the process by which literacy can affect health outcomes is not well understood. We and others have hypothesized that literacy's relationship to low health knowledge leads to less effective or inconsistent self-care behaviors and worse outcomes. Predictors of behaviors such as self-efficacy may be related to literacy and could mediate the relationship between literacy and health behaviors ${ }^{12,13}$. 
$\mathrm{HF}$ is an excellent model for studying the literacy-outcome relationship because of the complexity and central role of selfcare for attaining optimal outcomes. The demands of understanding HF physiology, daily monitoring of weight and symptoms, adherence to complex regimens, and effective utilization of health care may make HF self-care difficult in those with limited literacy ${ }^{14}$. Previous research suggests that HF patients with low literacy have less knowledge about their disease and that low literacy may make informed self-care decisions difficult ${ }^{15-17}$. Low literacy may influence the learning of essential HF self-care skills leading to more HF exacerbations, higher burden of symptoms, poorer $\mathrm{QOL}$, and increased risk of hospitalization and death ${ }^{13,14}$. However, the relationship from literacy to poor HF outcomes remains hypothesized with little empirical evidence to support the connection. The purpose of this paper is to explore the cross-sectional relationship between literacy and HF-related quality of life (HFGOL) and understand potential mediators of that relationship including HF-related knowledge, self-efficacy, and self-care behaviors among patients enrolled in a randomized trial of self-care training. We hypothesized that there would be a direct relationship between literacy and HFGOL and that relationship would be mediated by knowledge, self-efficacy, and selfcare behaviors.

\section{METHODS}

To examine the literacy-HFQOL relationship, we analyzed baseline data collected for a randomized controlled trial of intensive HF self-management education. Details regarding the study participant eligibility, recruitment procedures, and data collection processes have been previously described and are summarized here ${ }^{18}$

\section{Participants}

Study participants were recruited from 2007 to 2009 from university-affiliated General Internal Medicine and Cardiology clinics at four sites: University of North Carolina (UNC), Northwestern, San Francisco General Hospital, and Olive View-UCLA Medical Center. To be included in the study, each participant required a diagnosis of $\mathrm{HF}$, New York Heart Association (NYHA) class II-IV symptoms in the past 6 months, current use of a loop diuretic medication, and an absence of cognitive impairment ${ }^{19}$. Potential participants were approached at regular outpatient appointments. Those agreeing to participate provided informed consent. Subsequently, the research assistant (RA) administered baseline questionnaires (described below). The study protocol was approved by the Institutional Review Board Human Subjects Committee at all sites.

\section{Measures}

Background Information. During baseline interviews, we obtained race/ethnicity, insurance status, household income, subjective socioeconomic status ${ }^{20}$, years of education, and medication use through patient interviews. Subjective socioeconomic status (SES) is the respondent's assessment of his or her position in society relative to others based on wealth, educational attainment, and job-related respect ${ }^{21}$. We also collected age, gender, co-morbid conditions, prescribed medications, and diagnostic lab test and echocardiogram results from the medical chart. HF severity was assessed by interview and categorized by NYHA class ${ }^{22}$. All interviews were conducted verbally in the language of choice (English or Spanish) by a trained RA fluent in the language. All items that had not previously been administered in Spanish were translated by native Spanish speakers by forward and back translation.

Literacy. Literacy was measured with the short-Test of Functional Health Literacy in Adults (TOFHLA) in either English or Spanish. The TOFHLA is a 36-item, 7-minute timed test of reading comprehension, and is a reliable, validated measure of literacy in the health care context ${ }^{23,24}$. Each participant's literacy level was categorized as either inadequate/marginal (0-22 correct answers), which we refer to as "low literacy," or adequate literacy (23-36 correct answers).

\section{Outcome Measures}

HFQOL. Our main outcome of interest was patient-reported HFQOL. HFGOL was assessed at baseline using the Improving Chronic Illness Care Evaluation (ICICE) Heart Failure Symptom Scale (HFSS) which was adapted for phone interview from the Minnesota Living with Heart Failure Questionnaire and other $\mathrm{HF}$ health status scales ${ }^{25}$. The HFSS is a validated instrument that consists of seven questions with a five-point response scale, but is transformed to a 100-point scale with 100 representing the best symptom profile. The frame of reference for all items was the past 4 weeks. The HFSS shows high reliability and correlation with the SF-12 Physical Health Summary Scale $^{25}$. In our study, a difference of 14 points on a 0-100 scale is roughly equivalent to a change in one level of NYHA classification. Although the HFSS is a newer scale and has less validity data collected on its use, precursor instruments such as the Minnesota Living with Heart Failure Questionnaire have demonstrated responsiveness in numerous clinical trials of interventions to reduce hospitalization and mortality ${ }^{26-30}$

HF Knowledge and Self Care Behaviors. HF knowledge and self-care behaviors were assessed using an adapted version of the ICICE telephone survey ${ }^{31}$. HF-related knowledge questions included general $\mathrm{HF}$ questions, such as the definition of $\mathrm{HF}$, how often someone with $\mathrm{HF}$ should weigh himself/herself, and signs of $\mathrm{HF}$, with total scores ranging from $0-8$. Salt intake questions included which foods contain a lot of salt, what is a safe amount of salt, and why salt is bad for someone with $\mathrm{HF}$, with a total score ranging from 0-10. HF self-care behaviors included monitoring weight, knowing the appropriate responses to deal with a weight increase, exercising, and cutting back on salt (behavior scale range 0-10).

Self-Efficacy Scale. Self-efficacy, the patient's perceived ability to do the things required to manage his or her $\mathrm{HF}$ or perform HF-related behaviors, was measured with a 10-item scale 
developed for this trial. Items ask how sure patients are that they can: (1) tell whether their HF is getting worse; (2) eat foods that are low in salt; and (3) can explain their symptoms to their doctor. Respondents used a 10-point response scale ranging from 1 (not sure) to 10 (absolutely sure). We created a selfefficacy score calculated as the sum of items (Cronbach's alpha $=0.7275$ ). Thus, the self-efficacy score could range from 10-100.

Data Analysis. We examined baseline differences between low and adequate literacy groups using t-tests for continuous outcomes and chi-squared tests for categorical outcomes. We performed multivariate linear regression analyses to estimate the associations of literacy and HF symptoms, knowledge, selfefficacy, and behaviors, while controlling for the following potential confounders: race, ethnicity, age, sex, an indicator for whether the subject was ever uninsured in the previous 24 months, and subjective socioeconomic position. We did not include education in the models because of the intrinsic bidirectional relationship between education and literacy ${ }^{32}$. We chose to use subjective SES rather than income in the model because of the large number of respondents of retirement age who reported very low income, but have wealth we did not measure. Of the 605 patients, 18 did not answer the socioeconomic question and an additional two patients omitted answers to two or more items in the HFSS. These 20 observations were omitted from the multivariate analysis, leaving a final sample of 585 .

Structural Equation Models. We also assessed whether general HF knowledge, salt knowledge, self-care behaviors, and selfefficacy mediated the relationship between literacy and HFQOL. To do so, we developed a path model and estimated the relationships using structural equation modeling (SEM) and calculated estimated total, direct, and indirect standardized effects of the model for low literacy and SES on the five measures of interest.

Our model was specified based on previous research and hypotheses on the relationship between low literacy and health outcomes from the framework of social cognitive theory ${ }^{33-35}$. Low literacy was specified as being associated with various sociodemographic factors (gender, race/ethnicity, age, gap in insurance coverage) as well as SES. Low literacy was hypothesized to be associated with lower general $\mathrm{HF}$ knowledge and lower salt knowledge, which were expected to be associated with lower self-efficacy, worse self-care, and ultimately lower HFGOL. Direct effects (such as knowledge on self-care and HFGOL and self-efficacy on HFQOL) were also included in the model. Because low literacy may be a marker for SES which may affect these outcomes, we allowed SES to have identical paths as low literacy. If literacy is a noisy measure of SES, then standard theory suggests that low literacy would likely have weaker results than SES. Model fit was assessed using four statistics: an adjusted goodness of fit index (AGFI) greater than 0.90, root mean square error of approximation (RMSEA) less than 0.05, and normed fit index (NFI) greater than 0.90 , along with a chi-square $\mathrm{p}$-value $>0.05$ 34. Because the variables were not multivariate-normally distributed, we assessed statistical significance by bootstrapping with 1000 replications and employing maximum likelihood estimation methods, which has been shown to perform comparably to generalized least squares and better than weighted least squares in such contexts ${ }^{36}$. All SEM was performed using Amos 18's bootstrapping option to bootstrap the data to model the distribution of the indirect effects. We assessed statistical significance using the reported bias-corrected percentiles (AMOS Development Corporation: Spring House, PA).

\section{RESULTS}

\section{Participant Characteristics}

We enrolled 605 participants in total (Fig. 1). In general, the sample was ethnically diverse, had low income and education, and varied in HF symptoms (Table 1). The mean score on the TOFHLA was 24.2 with $37 \%$ categorized as having low literacy (score $<23$ on TOFHLA). Compared to those with adequate literacy, those with low literacy tended to be: older, ethnic minorities, male, speak Spanish, less likely to have completed high school, and have annual household incomes under $\$ 15,000$ (Table 1). People with low literacy also reported lower subjective SES on the 10-rung ladder question compared with people with adequate literacy.

HF Symptoms. In the overall sample, 31\% had Class III or IV symptoms at enrollment (Table 1). Those in the adequate literacy group were less likely to have NYHA class III or IV than those in the low literacy group ( $26 \%$ vs. $40 \%, \mathrm{p}<0.001)$. Mean score on the HFSS (HF-related symptoms) in the overall sample was $60.7 \pm 21.9$ (Table 2). Those with adequate literacy had better HFSS scores compared to those with low literacy $(63.9 \pm 22.1$ vs. $55.4 \pm 20.6$, adjusted difference 7.20 , [CI 11.13, 2.93]).

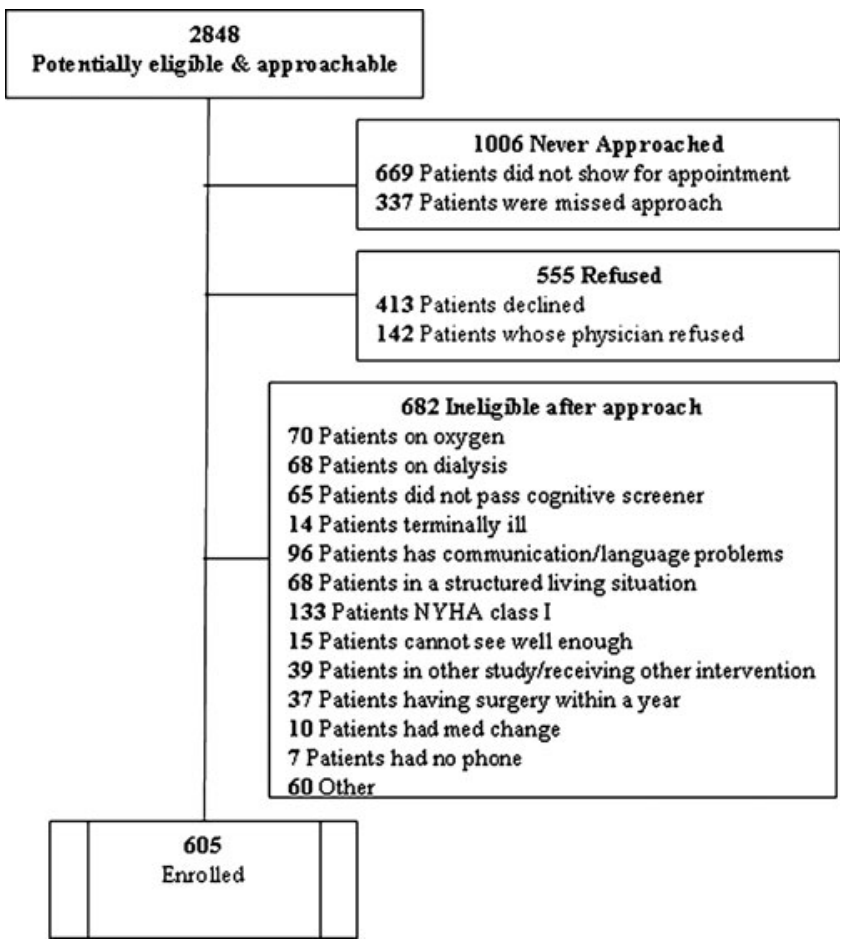

Figure 1. Recruitment and enrollment in the clinical trial. 
Table 1. Baseline Demographic and Clinical Characteristics and P values for Comparison of Low to Adequate Literacy

\begin{tabular}{|c|c|c|c|c|}
\hline & \multirow{2}{*}{$\frac{\text { Overall Sample }}{\mathrm{N}(\%) \text { or Mean } \pm \mathrm{SD}}$} & \multirow{2}{*}{$\begin{array}{l}\text { Adequate Literacy (TOFHLA }>=23 \text { ) } \\
\mathrm{N}(\%) \text { or Mean } \pm \text { SD }\end{array}$} & \multirow{2}{*}{$\begin{array}{l}\text { LowLiteracy (TOFHLA }<23 \text { ) } \\
\mathrm{N}(\%) \text { or Mean } \pm \mathrm{SD}\end{array}$} & \multirow[t]{2}{*}{$\mathbf{P}$} \\
\hline & & & & \\
\hline Size & 605 & 380 & 225 & \\
\hline \multicolumn{5}{|l|}{ Demographics } \\
\hline Literacy (TOFHLA) & $24.2 \pm 12.3$ & $32.7 \pm 3.7$ & $9.9 \pm 7.5$ & \\
\hline \multicolumn{5}{|l|}{ Site } \\
\hline UNC & $216(36)$ & $145(38)$ & $71(32)$ & \\
\hline NU & $166(27)$ & $143(38)$ & $23(10)$ & \\
\hline UCSF & $148(24)$ & $61(16)$ & 87 (39) & \\
\hline UCLA & $75(12)$ & $31(8)$ & $44(20)$ & \\
\hline Age & $60.7 \pm 13.1$ & $58.7 \pm 13.1$ & $64.2 \pm 12.4$ & $\mathrm{P}<0.001$ \\
\hline \multicolumn{4}{|l|}{ Race/Ethnicity } & $\mathrm{P}<0.001$ \\
\hline White NH & 233 (39) & $190(50)$ & 43 (19) & \\
\hline Hispanic & $97(16)$ & $29(8)$ & $68(30)$ & \\
\hline African American & $232(38)$ & $137(36)$ & $95(42)$ & \\
\hline Other & $41(7)$ & $22(6)$ & $19(8)$ & \\
\hline Missing & $2(0)$ & $2(1)$ & $0(0)$ & \\
\hline Gender: Male & $314(52)$ & $187(49)$ & $127(56)$ & $P=0.09$ \\
\hline \multirow{2}{*}{\multicolumn{4}{|c|}{ Income Level, \$ }} & $P<0.001$ \\
\hline & & & & $\mathrm{P}<0.001$ \\
\hline$<15,000$ & $305(52)$ & $151(41)$ & $154(71)$ & \\
\hline $15,000-24,999$ & $92(16)$ & $61(16)$ & $31(14)$ & \\
\hline $25,000-40,000$ & $67(11)$ & $51(14)$ & $16(7)$ & \\
\hline$>40,000$ & $125(21)$ & $108(29)$ & $17(8)$ & \\
\hline \multicolumn{4}{|l|}{ Education Level } & $\mathrm{P}<0.001$ \\
\hline$<12$ th grade & $160(26)$ & $46(12)$ & $114(51)$ & \\
\hline High School & $177(29)$ & $104(27)$ & $73(32)$ & \\
\hline Some college & $140(23)$ & $118(31)$ & $22(10)$ & \\
\hline College graduate or greater & $128(21)$ & $112(29)$ & $16(7)$ & \\
\hline Subjective Socioeconomic Status & $4.8 \pm 2.5$ & $5.4 \pm 2.4$ & $3.7 \pm 2.3$ & $\mathrm{P}<0.001$ \\
\hline \multicolumn{4}{|l|}{ Insurance } & $\mathrm{P}<0.001$ \\
\hline Medicare Only & $65(11)$ & $34(9)$ & $31(14)$ & \\
\hline Medicare \& Medicaid & $104(17)$ & 49 (13) & $55(24)$ & \\
\hline Medicare \& Private & $129(21)$ & $90(24)$ & $39(17)$ & \\
\hline Medicaid & $151(25)$ & $83(22)$ & $68(30)$ & \\
\hline Private & 77 (13) & $71(19)$ & $6(3)$ & \\
\hline \multirow[t]{3}{*}{ Uninsured } & 79 (13) & $53(14)$ & $26(12)$ & \\
\hline & Overall Sample & Adequate Literacy (TOFHLA $>=23$ ) & Low Literacy (TOFHLA <23) & $\mathrm{P}$ \\
\hline & $\mathrm{N}(\%)$ or Mean $\pm \mathrm{SD}$ & $\mathrm{N}(\%)$ or Mean $\pm \mathrm{SD}$ & $\mathrm{N}(\%)$ or Mean $\pm \mathrm{SD}$ & \\
\hline \multicolumn{5}{|l|}{ Clinical Characteristics } \\
\hline \multicolumn{4}{|l|}{ NYHA Class } & $\mathrm{P}<0.001$ \\
\hline I & $114(19)$ & $83(22)$ & $31(14)$ & \\
\hline II & $304(50)$ & $200(53)$ & $104(46)$ & \\
\hline III & $118(20)$ & $64(17)$ & $54(24)$ & \\
\hline IV & $69(11)$ & $33(9)$ & $36(16)$ & \\
\hline Systolic Dysfunction: & $355(60)$ & $226(61)$ & $129(58)$ & $\mathrm{P}=0.51$ \\
\hline \multicolumn{5}{|l|}{ Ejection fraction $<0.45$} \\
\hline Systolic BP (mm/Hg) & $\begin{array}{l}124.8 \pm 22.7 \\
(\mathrm{~N}=604)\end{array}$ & $\begin{array}{l}122.0 \pm 22.7 \\
(\mathrm{~N}=380)\end{array}$ & $\begin{array}{l}129.4 \pm 22.0 \\
(\mathrm{~N}=224)\end{array}$ & $\mathrm{P}<0.001$ \\
\hline Diastolic BP (mm/Hg) & $\begin{array}{l}71.3 \pm 12.9 \\
(\mathrm{~N}=604)\end{array}$ & $\begin{array}{l}71.6 \pm 12.6 \\
(\mathrm{~N}=380)\end{array}$ & $\begin{array}{l}70.7 \pm 13.3 \\
(\mathrm{~N}=224)\end{array}$ & $\mathrm{P}=0.38$ \\
\hline Body Mass index & $33.1 \pm 8.9$ & $33.1 \pm 8.9$ & $33.1 \pm 8.8$ & $\mathrm{P}=0.99$ \\
\hline & $(\mathrm{N}=602)$ & $(\mathrm{N}=378)$ & $(\mathrm{N}=224)$ & \\
\hline Creatinine level & $1.26 \pm 0.54$ & $1.26 \pm 0.54$ & $1.26 \pm 0.55$ & $\mathrm{P}=0.93$ \\
\hline & $(\mathrm{N}=604)$ & $(\mathrm{N}=379)$ & $(\mathrm{N}=225)$ & \\
\hline Diabetes & $290(48)$ & $168(44)$ & $122(54)$ & $\mathrm{P}=0.02$ \\
\hline Hypertension & $513(85)$ & $297(78)$ & $216(96)$ & $\mathrm{P}<0.001$ \\
\hline Previous MI or angina & $230(38)$ & $122(32)$ & $108(48)$ & $\mathrm{P}<0.001$ \\
\hline PHQ Score & $7.4 \pm 5.4$ & $7.2 \pm 5.5$ & $7.9 \pm 5.3$ & $\mathrm{P}=0.14$ \\
\hline Current smoker & $96(16)$ & $67(18)$ & $29(13)$ & $\mathrm{P}=0.12$ \\
\hline Medication History & & & & \\
\hline ACE-I & $391(66)$ & $245(66)$ & $146(65)$ & $\mathrm{P}=0.96$ \\
\hline $\mathrm{ARB}$ & $120(20)$ & $72(19)$ & $48(21)$ & $\mathrm{P}=0.49$ \\
\hline ACE-I or ARB & $497(82)$ & $310(82)$ & $187(83)$ & $P=0.63$ \\
\hline Beta blocker & $492(82)$ & $313(83)$ & $179(80)$ & $\mathrm{P}=0.38$ \\
\hline Spironolactone & $166(27)$ & $119(31)$ & $47(21)$ & $\mathrm{P}=0.01$ \\
\hline
\end{tabular}

NH=Non-Hispanic, TOFHLA=Test of Functional Health Literacy in Adults

$A C E-I=A c e-i n h i b i t o r, A R B=$ angiotensin receptor blocker, $B P=$ blood pressure, $M I=$ myocardial infarction, $N Y H A=$ New York Heart Association, $P H Q=P a t i e n t$ Health Questionnaire, TOFHLA=Test of Functional Health Literacy in Adults 
Table 2. Association Between Adequate Literacy and Heart Failure Symptom Scale, Heart Failure Knowledge, Salt Knowledge, and Selfefficacy Scales After Adjustment for Sociodemographic Characteristics

\begin{tabular}{|c|c|c|c|c|c|}
\hline & Overall Sample & $\begin{array}{l}\text { Adequate Literacy } \\
\text { (TOFHLA > =23) }\end{array}$ & $\begin{array}{l}\text { Low Literacy } \\
\text { (TOFHLA <23) }\end{array}$ & $\begin{array}{l}\text { Unadjusted } \\
\text { Difference }\end{array}$ & $\begin{array}{l}\text { Adjustedt } \\
\text { Difference }\end{array}$ \\
\hline & $\begin{array}{l}\text { Mean } \pm \text { SD Median } \\
\text { (interquartile) }\end{array}$ & $\begin{array}{l}\text { Mean } \pm \text { SD Median } \\
\text { (interquartile) }\end{array}$ & $\begin{array}{l}\text { Mean } \pm \text { SD Median } \\
\text { (interquartile) }\end{array}$ & $\begin{array}{l}\text { Coefficient } \\
(95 \% \mathrm{Cl})\end{array}$ & $\begin{array}{l}\text { Coefficient } \\
(95 \% \mathrm{Cl})\end{array}$ \\
\hline \multirow[t]{2}{*}{ HFGOL } & $60.7 \pm 22.1$ & $63.9 \pm 22.2$ & $55.3 \pm 20.8$ & $8.52^{* *}(4.92,12.13)$ & $8.20^{* *}(4.14,12.27)$ \\
\hline & $60.7[46.4,78.6]$ & $64.3[46.4,82.1]$ & $57.1[42.9,67.9]$ & $7.14^{* *}(3.57,14.29)$ & $7.14 *(1.26,13.02)$ \\
\hline \multirow[t]{2}{*}{ HF Knowledge } & $6.1 \pm 1.8$ & $6.5 \pm 1.6$ & $5.5 \pm 1.9$ & $1.08^{* *}(0.80,1.36)$ & $0.72^{* *}(0.39,1.04)$ \\
\hline & $7[5.0,8.0]$ & $7[6.0,8.0]$ & $6[4.0,7.0)$ & $1^{* *}(1.00,2.00)$ & $1^{* *}(0.72,1.28)$ \\
\hline \multirow[t]{2}{*}{ Salt Knowledge } & $7.5 \pm 1.5$ & $7.8 \pm 1.3$ & $7.0 \pm 1.7$ & $0.77^{* *}(0.53,1.02)$ & $0.63^{* *}(0.34,0.91)$ \\
\hline & $8[7.0,9.0]$ & $8[7.0,9.0]$ & $7[6.0,8.0]$ & $1^{* *}(0.00,1.00)$ & $1(-1.55,3.55)$ \\
\hline \multirow[t]{2}{*}{ Self-Care Behavior } & $4.5 \pm 2.1$ & $4.9 \pm 2.0$ & $3.8 \pm 2.0$ & $1.11^{* *}(0.78,1.44)$ & $0.81^{* *}(0.43,1.19)$ \\
\hline & $4[3.0,6.0]$ & $5[3.0,6.0]$ & $4[2.0,5.0]$ & $1^{* *}(1.00,2.00)$ & $0.80^{* *}(.25,1.35)$ \\
\hline \multirow[t]{2}{*}{ Self-Efficacy Scale } & $78.3 \pm 14.3$ & $81.0 \pm 12.9$ & $73.7 \pm 15.4$ & $7.29^{* *}(4.98,9.59)$ & $6.09 * *(3.44,8.74)$ \\
\hline & $81[70.0,89.0]$ & $84[74.0,90.0]$ & $75[64.0,85.0]$ & $9 * *(5.00,12.00)$ & $7.25^{* *}(3.69,10.81)$ \\
\hline
\end{tabular}

HF=heart failure; HFQOL=Heart failure-related quality of life; TOFHLA=Test of Functional Health Literacy in Adults

$\dagger$ Adjusted for race/ethnicity, age, insurance coverage, previously uninsured, and subjective socioeconomic position

$N=585$. Confidence intervals for unadjusted difference in medians use bootstrapping; adjusted difference for second row uses median regression

* significant at 5\%; ** significant at 1\%

General HF Knowledge. Patients with adequate literacy had higher general HF knowledge (mean score 6.2 vs. 5.5, adjusted difference 0.63 [CI 0.97, 0.29]) (Table 2). Table 3 presents the results for each general knowledge question.

Salt Knowledge. Salt knowledge scores were higher for those with adequate literacy (8.2 vs. 7.5 , adjusted difference 0.54 [CI $0.85,0.23]$ ) (Table 2). Specific item responses are shown in Table 3.
Self-Efficacy. Participants with adequate literacy had higher overall self-efficacy (5.0 vs. 4.1, adjusted difference 0.99 [CI 1.55, 0.43]).

Self-Care Behaviors. Participants with adequate literacy reported higher behavior scores than those with low literacy (mean score 5.3 vs. 4.2, adjusted difference 0.59 [CI 0.96, 0.22]) (Table 2). More adequate literacy participants reported having a scale at home $(58 \%$ vs.

Table 3. Baseline Heart Failure General and Salt Knowledge According to Literacy Level

\begin{tabular}{|c|c|c|c|c|}
\hline & \multirow{2}{*}{$\begin{array}{l}\text { Overall } \\
\text { Sample } \\
\mathrm{N}(\%)\end{array}$} & \multirow{2}{*}{$\begin{array}{l}\text { Adequate Literacy } \\
\text { (TOFHLA >=23) } \\
\mathrm{N}(\%)\end{array}$} & \multirow{2}{*}{$\begin{array}{l}\text { Low Literacy } \\
\text { (TOFHLA<23) } \\
\mathrm{N}(\%)\end{array}$} & \multirow[t]{2}{*}{$\mathbf{P}$} \\
\hline & & & & \\
\hline \multicolumn{5}{|l|}{ Heart Failure General Knowledge (Correct Response) } \\
\hline $\begin{array}{l}\text { 1. How often someone with HF should weigh himself/herself? } \\
\text { (Every day) }\end{array}$ & $335(55)$ & $244(64)$ & $91(40)$ & $\mathrm{P}<0.001$ \\
\hline $\begin{array}{l}\text { 2. What is the best definition of HF? (HF means that your heart } \\
\text { is not pumping blood as well as it should) }\end{array}$ & $442(73)$ & 315 (83) & $127(56)$ & $\mathrm{P}<0.001$ \\
\hline 3. Is shortness of breath a sign of heart failure? (Y) & $474(78)$ & $309(81)$ & $165(73)$ & $\mathrm{P}=0.02$ \\
\hline 4. Is swelling of legs or ankles a sign of heart failure? (Y) & $502(83)$ & $334(88)$ & $168(75)$ & $\mathrm{P}<0.001$ \\
\hline 5. Is waking up at night short of breath a sign of heart failure? (Y) & $502(83)$ & $325(86)$ & $177(79)$ & $\mathrm{OP}=0.03$ \\
\hline 6. Is feeling more tired than usual a sign of heart failure? $(\mathrm{Y})$ & $499(82)$ & $326(86)$ & $173(77)$ & $P=0.01$ \\
\hline 7. Is weight gain a sign of heart failure? $(\mathrm{Y})$ & $389(64)$ & $273(72)$ & $116(52)$ & $\mathrm{P}<0.001$ \\
\hline $\begin{array}{l}\text { 8. Is it safe for someone with heart failure to do light exercise } \\
\text { like walking? }(\mathrm{Y})\end{array}$ & $574(95)$ & $361(95)$ & $213(95)$ & $\mathrm{P}=0.86$ \\
\hline \multicolumn{5}{|l|}{ Heart Failure Salt Knowledge (Correct Response) } \\
\hline \multicolumn{5}{|l|}{ Do the following foods contain a lot of salt? (\% correct) } \\
\hline 1. Hot dogs $(\mathrm{Y})$ & $552(91)$ & $358(94)$ & $194(86)$ & $\mathrm{P}<0.001$ \\
\hline 2. Orange juice $(\mathrm{N})$ & $413(68)$ & $266(70)$ & $147(65)$ & $P=0.23$ \\
\hline 3. Canned vegetables $(\mathrm{Y})$ & $515(85)$ & $345(91)$ & $170(76)$ & $\mathrm{P}<0.001$ \\
\hline 4. Bananas $(\mathrm{N})$ & $512(85)$ & $329(87)$ & $183(81)$ & $\mathrm{P}=0.08$ \\
\hline 5. Cheese (Y) & $471(78)$ & $292(77)$ & $179(80)$ & $\mathrm{P}=0.44$ \\
\hline 6. Eggs (N) & $476(79)$ & $300(79)$ & $176(78)$ & $P=0.83$ \\
\hline 7. Ranch salad dressing $(\mathrm{Y})$ & $487(80)$ & $321(84)$ & $166(74)$ & $\mathrm{P}=0.001$ \\
\hline 8. Is sodium another name for salt? $(\mathrm{Y})$ & $568(94)$ & 374 (99) & $193(86)$ & $\mathrm{P}<0.001$ \\
\hline 9. Is $140 \mathrm{MG} /$ serving a safe amount of sodium? (Y) & $56(9)$ & $35(9)$ & $21(9)$ & $P=0.96$ \\
\hline $\begin{array}{l}\text { 10. Why is salt bad for someone with HF? c. salt causes your } \\
\text { body to hold on to water and swell up (Correct) }\end{array}$ & $509(84)$ & $352(93)$ & $157(70)$ & $\mathrm{P}<0.001$ \\
\hline
\end{tabular}


Table 4. Heart Failure Self Care Behavior Items According to Literacy Level

\begin{tabular}{|c|c|c|c|c|}
\hline & $\begin{array}{l}\text { O v e r a I I } \\
\text { Sample }\end{array}$ & $\begin{array}{l}\text { Adequate Literacy } \\
\text { (TOFHLA }>=23 \text { ) }\end{array}$ & $\begin{array}{l}\text { Low Literacy } \\
\text { (TOFHLA<23) }\end{array}$ & $\mathbf{P}$ \\
\hline & $\mathrm{N}(\%)$ & $\mathrm{N}(\%)$ & N (\%) & \\
\hline \multicolumn{5}{|l|}{ Heart Failure Self Care (Correct Response) } \\
\hline 1. Do you know what your target weight is? $(\mathrm{Y})$ & $102(17)$ & $65(17)$ & $37(16)$ & $\mathrm{P}=0.83$ \\
\hline 2. Do you have a scale at home that works correctly? (Y) & $318(53)$ & $222(58)$ & $96(43)$ & $\mathrm{P}<0.001$ \\
\hline 3. Do you weigh yourself every day? $(\mathrm{Y})$ & $157(26)$ & $122(32)$ & $35(16)$ & $\mathrm{P}<0.001$ \\
\hline $\begin{array}{l}\text { 4. During the last } 6 \text { months have you taken specific steps to eat foods that } \\
\text { are low in salt? }(\mathrm{Y})\end{array}$ & $488(81)$ & $318(84)$ & $170(76)$ & $\mathrm{P}=0.01$ \\
\hline $\begin{array}{l}\text { 5. Have you been very successful at sticking to your plans to eat foods that } \\
\text { are low in salt? }(\mathrm{Y})\end{array}$ & $149(25)$ & $99(26)$ & $50(22)$ & $\mathrm{P}=0.29$ \\
\hline $\begin{array}{l}\text { 6. Has anyone ever taught you to change the number of "water pills" you } \\
\text { take? (Y) }\end{array}$ & $246(41)$ & $170(45)$ & $76(34)$ & $\mathrm{P}=0.01$ \\
\hline 7. Do you change the number of water pills you take when you need to? $(\mathrm{Y})$ & $244(40)$ & $180(47)$ & $64(28)$ & $\mathrm{P}<0.001$ \\
\hline $\begin{array}{l}\text { 8. Do you have a plan prepared by your heart failure team that tells you what } \\
\text { to do if your heart failure symptoms get worse? (Y) }\end{array}$ & $300(50)$ & $190(50)$ & $110(49)$ & $P=0.79$ \\
\hline $\begin{array}{l}\text { 9. What do you do if your weight goes up by } 4 \text { pounds? (Acceptable } \\
\text { responses: 'cut back on salt', 'take an extra water pill', or 'call your provider') }\end{array}$ & $388(64)$ & $277(73)$ & $111(49)$ & $\mathrm{P}<0.001$ \\
\hline 10. Do you walk or exercise 3 or more days per week? & $346(57)$ & $233(61)$ & $113(50)$ & $\mathrm{P}=0.01$ \\
\hline
\end{tabular}

TOFHLA=Test of Functional Health Literacy in Adults

$43 \%, \mathrm{p}<0.001)$ and reported weighing themselves everyday (32\% vs. $16 \%, \mathrm{p}<0.001)$ compared to those with low literacy. More patients with adequate literacy eat foods that are low in salt than those with low literacy (84\% vs. $76 \%, \mathrm{p}=0.014)$. More participants in the adequate literacy group were taught to manage their diuretic $(45 \%$ vs. $34 \%$, $\mathrm{p}=0.008)$ and actually performed this behavior $(47 \%$ vs. $28 \%, \mathrm{p}<0.001)$ compared to those in the low literacy group. Moreover, more patients with adequate literacy knew what to do if their weight went up 4 pounds compared with patients with low literacy $(73 \%$ vs. $49 \%$, p<0.001) Table 4.

\section{Structural Equation Models}

Figure 2 presents the standardized coefficient estimates from the SEM analysis. Post-hoc analysis revealed two paths that were not initially specified: a gap in insurance coverage was associated with statistically significant higher knowl-

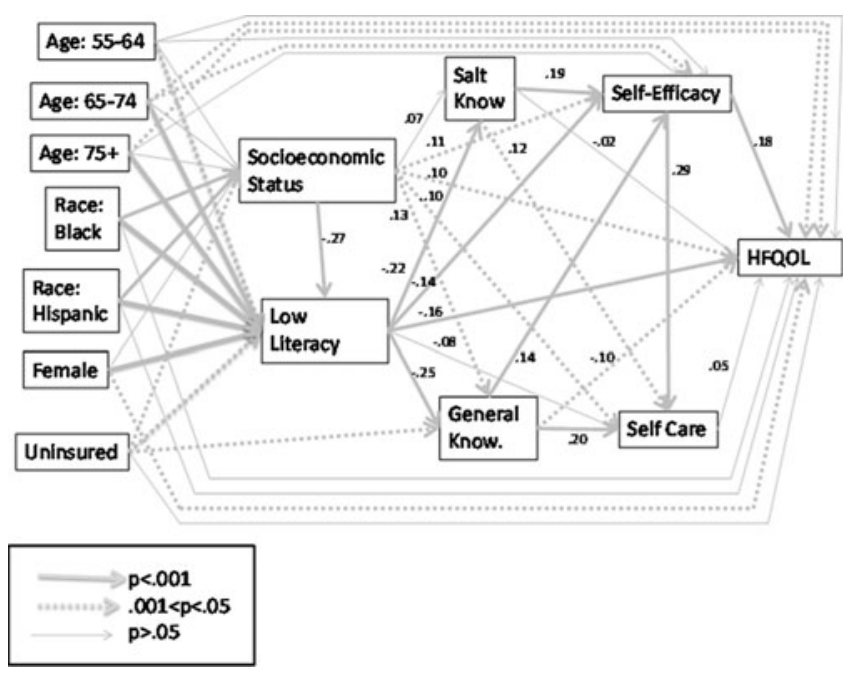

Figure 2. Structural equation model results. edge and subjects between the ages of 65 and 74 had higher self-efficacy than subjects below the age of 55. After including these additional pathways, the model passed all four of our tests for model fit (AGFI $=0.962$, RMSEA $=0.029$, chi-square $\mathrm{p}$-value $=0.053$, and NFI $=0.970$ ). Most coefficients were of the hypothesized direction; low literacy was associated with poorer $\mathrm{HF}$ - specific (standardized coefficient -0.25, $\mathrm{p}=0.003)$ and salt knowledge $(-0.22, \mathrm{p}=0.003)$, lower self-efficacy $(-0.143, \mathrm{p}=0.003)$ and worse HFQOL $(-0.16, p=0.003)$, although it did not have a direct effect on self-care $(-0.08, p=0.09)$. Low literacy had important effects independent of SES, demonstrating that low-literacy is a separate domain from SES.

Table 5 presents total effects, direct effects, and indirect effects. Direct effects are the estimated regression coefficients. Indirect effects are the products of the estimated coefficients along the paths. The total effect is the sum of the direct effect and the indirect effect. All estimated effects for low literacy were statistically significant at conventional levels. The indirect effect of low literacy on self care was approximately two-thirds of the total effect, which was not

Table 5. Estimated Total, Direct, and Indirect Effects of Low Literacy and Socioeconomic Status on Mediators of Heart Failure-Related Quality of Life

\begin{tabular}{llll}
\hline \hline & Total Effects & Direct Effects & Indirect Effects \\
\hline Low Literacy & & & \\
Salt Knowledge & $-0.219^{* *}$ & $-0.219^{* *}$ & - \\
General Knowledge & $-0.248^{* *}$ & $-0.248^{* *}$ & - \\
Self-Efficacy & $-0.220^{* *}$ & $-0.143^{* *}$ & $-0.077^{* *}$ \\
Self Care & $-0.214^{* *}$ & -0.076 & $-0.138^{* *}$ \\
HFGOL & $-0.177^{* *}$ & $-0.157^{* *}$ & -0.020 \\
Socioeconomic Status & & & \\
Salt Knowledge & $0.129^{* *}$ & 0.070 & $0.059^{* *}$ \\
General Knowledge & $0.192^{* *}$ & $0.125^{* *}$ & $0.067^{* *}$ \\
Self-Efficacy & $0.205^{* *}$ & $0.114^{* *}$ & $0.091^{* *}$ \\
Self Care & $0.227^{* *}$ & $0.095^{*}$ & $0.133^{* *}$ \\
HFGOL & $0.171^{* *}$ & $0.103^{*}$ & $0.068^{* *}$ \\
\hline
\end{tabular}

$\mathrm{HFBOL}=$ Heart failure-related quality of life

**: significant at 1\%; *: significant at 5\%, based on 1000 replications

"- "denotes no indirect effect exists 
statistically significant. The indirect effect of low literacy on HFQOL is small relative to the total effect (approximately $10 \%$ ) and is not statistically significant. Thus, the effect of low literacy on HFGOL is not mediated by the four potential mediators, but the effect of low literacy on self care is completely mediated by general knowledge, salt knowledge, and self-efficacy; the non-significance of the path from selfcare to HFQOL reconciles these results.

\section{DISCUSSION}

In our cross-sectional analysis, low literacy was associated with worse HFGOL even after adjusting for potential confounders. Low literacy was also associated with lower general and salt-related HF knowledge, lower performance of self-care behaviors, lower self-efficacy, and lower HFQOL. Although, knowledge and self-efficacy predict self-care behaviors, these variables did not explain the literacy-related difference in HFGOL.

Several studies in different contexts have evaluated the relationship between literacy and symptoms or HRQOL ${ }^{14,37-}$ 43. In general, people with low literacy score worse on depression and functional status questionnaires for a variety of health conditions. One study that examined fatigue among cancer patients did not find a relationship between literacy and fatigue ${ }^{43}$. In our previous randomized trial testing a literacy sensitive self-care intervention in 123 patients with HF, we found modest, but non-statistically significant differences in HRQOL between literacy groups at baseline ${ }^{15}$. Our current study had substantially more power to detect a difference than the previous study. However, the relationship between literacy and symptoms or HRQOL is not completely consistent and will require ongoing investigation.

Our study revealed that those with low literacy have less knowledge about HF compared to those with adequate literacy. Several reports have found that patients with low literacy are significantly less likely to correctly answer questions about HF suggesting they may have insufficient knowledge to manage their disease $\mathrm{e}^{17,44,45}$. It has been suggested that low literacy may limit information retention, making the learning of essential HF self-care skills more difficult, leading to poorer health outcomes ${ }^{14}$.

We hypothesized that HF patients with low literacy would have lower knowledge, self-efficacy, and actual behaviors and these factors would mediate the relationship between literacy and HF-related symptoms. We confirmed our hypothesized relationship between low literacy and lower knowledge, selfefficacy, and self-care behaviors; however, our mediation analyses did not support our hypothesized pathway to the outcome. Although low literacy's effect on self-care was completely mediated, there was no mediating effect on HFQOL. This finding is largely consistent with a study in diabetes showing that knowledge, behavior, and self-efficacy did not mediate the relationship between literacy and glycemic control ${ }^{12}$. These results underscore the complicated relationship between literacy and outcomes and pose challenges to the traditional model of the hypothetical pathways between literacy and health outcomes $^{35,46}$.
It is possible that our measures of knowledge, behavior, and self-efficacy were not precise enough to detect a pathway between inadequate literacy and worse symptoms. These are difficult variables to measure, and no instrument can address all aspects of care for an individual. The influence of literacy may be so pervasive across all aspects of health behaviors that it will be difficult to identify the specific knowledge, behavior, or other variables that mediate the relationship between low literacy and worse health. Perhaps a more fundamental problem with this analysis is the cross-sectional nature of the data. It is possible that patients with more symptoms have received more education related to their HF. For example, patients with more severe HF may have more outpatient visits and hospitalizations, resulting in greater exposure to patient education programs and more knowledge. It is also possible that we have an incomplete model relating low literacy to HFGOL, and that other unmeasured factors are influential.

Our study has other limitations. Knowledge, behaviors, and symptoms were assessed using self-report measures administered orally. Hence, the items could be subject to a social desirability bias. Such an effect would likely bias the results toward the null for behavior and self-efficacy items. For knowledge, however, we would not expect such a bias. More participants with low literacy were enrolled from the California sites and may not be representative of all persons with low literacy. Finally, the multiple testing may have inflated type I error rates.

\section{CONCLUSION}

HF patients with low literacy had worse HF-related symptoms, knowledge, self-efficacy, and self-care behaviors. Self-care behaviors, as measured in this study, did not mediate the relationship between literacy and HFGOL.

Acknowledgements: This study was funded by the National Institute of Health/National Heart, Lung, and Blood Institute (NIH/ NHLBI) -University of North Carolina at Chapel Hill, Grant \# 5R01HL081257 (PI: Pignone, M.). This paper was presented as a poster at the Society of General Internal Medicine 33rd Annual Meeting in Minneapolis, in April, 2010.

\section{Conflict of Interest: None disclosed.}

Open Access: This article is distributed under the terms of the Creative Commons Attribution Noncommercial License which permits any noncommercial use, distribution, and reproduction in any medium, provided the original author(s) and source are credited.

Corresponding Author: Aurelia Macabasco-O'Connell, $P h D, R N$, ACNP; UCLA School of Nursing, University of California, Los Angeles, 700 Tiverton Ave., 4-242 Factor Blvd., Los Angeles, CA 90095, USA (e-mail: aoconnel@sonnet.ucla.edu).

\section{REFERENCES}

1. Klersy C, De Silvestri A, Gabutti G, et al. A meta-analysis of remote monitoring of heart failure patients. J Am Coll Cardiol. 2009;54:16831694. doi:10.1016/j.jacc.2009.08.017.

2. Kirsch IJA, Jenkins L, Kolstat A. Adult Literacy in America: A First Look at the Findings of the National Adult Literacy Survey. 3rd ed. Washington, DC: National Center for Education, US Department of Education; 2002 
3. Williams M, Baker D, Parker R, et al. Relationship of functional health literacy to patients' knowledge of their chronic disease: A study of patients with hypertension and diabetes. Arch Intern Med. 1998;158:166-172.

4. Williams MV, Baker DW, Honig EG, et al. Inadequate literacy is a barrier to asthma knowledge and self-care. Chest. 1998;114:1008-1015. doi: $10.1378 /$ chest. 114.4.1008.

5. Scott T, Gazmararian J, Williams $\mathbf{M}$, et al. Health literacy and preventive health care use among Medicare enrollees in a managed care organization. Med Care. 2002;40:395-404.

6. Baker DW, Parker RM, Williams MV, et al. Health literacy and the risk of hospital admission. J Gen Intern Med. 1998;13:791-798.

7. Baker DW, Gazmararian JA, Sudano J, et al. Health literacy and performance on the Mini-Mental State Examination. Aging Ment Health. 2002;6:22-29. doi:10.1080/13607860120101121.

8. Sudore R, Yaffe $\mathbf{K}$, Satterfield $\mathbf{S}$, et al. Limited literacy is associated with mortality in the elderly: the health, aging, and body composition study. J Gen Intern Med. 2006;21:806-812.

9. Baker D, Wolf $\mathbf{M}$, Feinglass $\mathbf{J}$, et al. Health literacy and mortality among elderly persons. Arch Intern Med. 2007;167:1503-1509.

10. Baker DW, Parker RM, Williams MV, et al. The relationship of patient reading ability to self-reported health and use of health services. Am J Public Health. 1997;87:1027-1030.

11. Baker D, Gazmararian J, Williams M, et al. Functional health literacy and the risk of hospital admission among Medicare managed care enrollees. Am J Public Health. 2002;92:1278-1283.

12. DeWalt DA, Boone RS. Pignone MP Literacy and its relationship with self-efficacy, trust, and participation in medical decision making. Am J Health Behav. 2007;31(Suppl 1):S27-35

13. Wolf MS, Davis TC, Osborn CY, et al. Literacy, self-efficacy, and HIV medication adherence. Patient Educ Couns. 2007;65:253-260.

14. Riegel B, Moser DK, Anker SD, et al. State of the science: promoting self-care in persons with heart failure: a scientific statement from the American Heart Association. Circulation. 2009;120:1141-1163. doi:10.1161/circulationaha.109.192628

15. Dewalt D, Malone R, Bryant M, et al. A heart failure self-management program for patients of all literacy levels: A randomized, controlled trial [ISRCTN11535170]. BMC Health Serv Res. 2006;6:30.

16. Laramee A, Morris N. Littenberg B Relationship of literacy and heart failure in adults with diabetes. BMC Health Serv Res. 2007;7:98.

17. Gazmararian JA, Williams MV, Peel J, et al. Health literacy and knowledge of chronic disease. Patient Educ Couns. 2003;51:267-275.

18. DeWalt D, Broucksou K, Hawk V, et al. Comparison of a one-time educational intervention to a teach-to-goal educational intervention for self-management of heart failure: design of a randomized controlled trial. BMC Health Serv Res. 2009;9:99.

19. Zuccala G, Pedone C, Cesari M, et al. The effects of cognitive impairment on mortality among hospitalized patients with heart failure. Am J Med. 2003;115:97-103.

20. Singh-Manoux A, Adler NE. Marmot MG subjective social status: its determinants and its association with measures of ill-health in the Whitehall II study. Soc Sci Med. 2003;56:1321-1333.

21. Adler, NSJ. The MacArthur Scale of Subjective Social Status Psychosocial Notebook. MacArthur Research Network on SES and Health. Accessed 11/4/2010. http://www.macses.ucsf.edu/research/psycho social/subjective.php)

22. Hunt SA, Abraham WT, Chin MH, et al. ACC/AHA 2005 Guideline update for the diagnosis and management of chronic heart failure in the adult: a report of the American College of Cardiology/American Heart Association Task Force on Practice Guidelines (Writing Committee to Update the 2001 Guidelines for the Evaluation and Management of Heart Failure): Developed in collaboration with the American College of Chest Physicians and the International Society for Heart and Lung Transplantation: Endorsed by the Heart Rhythm Society.2005; e154-235.

23. Parker RM, Baker DW, Williams MV, et al. The test of functional health literacy in adults: a new instrument for measuring patients' literacy skills. J Gen Intern Med. 1995;10:537-541.

24. Baker DW, Williams MV, Parker RM, et al. Development of a brief test to measure functional health literacy. Patient Educ Couns. 1999;38:33-42.
25. Faxon DP, Schwamm LH, Pasternak RC, et al. Improving quality of care through disease management: principles and recommendations from the American Heart Association's Expert Panel on Disease Management. Circulation. 2004;109:2651-2654. doi:10.1161/01.CIR. 0000128373.90851.7B

26. Harrison MB, Browne GB, Roberts J, et al. Quality of life of individuals with heart failure: a randomized trial of the effectiveness of two models of hospital-to-home transition. Med Care. 2002;40:271-282.

27. Kasper EK, Gerstenblith G, Hefter G, et al. A randomized trial of the efficacy of multidisciplinary care in heart failure outpatients at high risk of hospital readmission. J Am Coll Cardiol. 2002;39:471-480.

28. Doughty RN, Wright SP, Pearl A, et al. Randomized, controlled trial of integrated heart failure management. The Auckland Heart Failure Management Study. Eur Heart J. 2002;23:139-146. doi:10.1053/euhj.2001.2712.

29. Koelling TM, Johnson ML, Cody RJ, et al. Discharge education improves clinical outcomes in patients with chronic heart failure. Circulation. 2005;111:179-185. doi:10.1161/01.cir.0000151811.53450.b8.

30. Goldberg LR, Piette JD, Walsh MN, et al. Randomized trial of a daily electronic home monitoring system in patients with advanced heart failure: the Weight Monitoring in Heart Failure (WHARF) trial. Am Heart J. 2003; 146:705-712.

31. Baker DW, Brown J, Chan Ks, et al. A telephone survey to measure communication, education, self-management, and health status for patients with heart failure: the Improving Chronic Illness Care Evaluation (ICICE). J Card Fail. 2005;11:36-42.

32. DeWalt DA, Pignone MP. Reading is fundamental: the relationship between literacy and health. Arch Intern Med. 2005;165:1943-1944. doi:10.1001/archinte.165.17.1943.

33. Osborn CY, Cavanaugh K, Wallston KA, et al. Self-efficacy links health literacy and numeracy to Glycemic Control. $\mathrm{J}$ Health Commun: Int Perspect. 15:146-158.

34. Cho YI, Lee S-YD, Arozullah AM, et al. Effects of health literacy on health status and health service utilization amongst the elderly. Soc Sci Med. 2008;66:1809-1816.

35. Pignone MP, DeWalt DA. Literacy and health outcomes: is adherence the missing link? J Gen Intern Med. 2006;21:896-897.

36. Olsson UH, Foss T, Troye SV, et al. The performance of ML, GLS, and WLS estimation in structural equation modeling under conditions of misspecification and nonnormality. Struct Equ Modeling: A Multidisciplinary Journal. 2000;7:557-595.

37. Muir KW, Santiago-Turla C, Stinnett SS, et al. Health literacy and vision-related quality of life. Br J Ophthalmol. 2008;92:779-782. doi:10.1136/bjo.2007.134452.

38. Nokes KM, Coleman CL, Cashen M, et al. Health literacy and health outcomes in HIV seropositive persons. Res Nurs Health. 2007;30:620627

39. Mancuso CA, Rincon M. Impact of health literacy on longitudinal asthma outcomes. J Gen Intern Med. 2006;21:813-817.

40. Lincoln A, Paasche-Orlow MK, Cheng DM, Lloyd-Travaglini C, Caruso C, Saitz R, Samet H. Impact of health literacy on depressive symptoms and mental health-related: quality of life among adults with addiction. J Gen Intern Med. 2006;21:818-822.

41. Roth MT. Ivey JL Self-reported medication use in community-residing older adults: A pilot study. Am J Geriatr Pharmacother. 2005;3:196-204.

42. Bennett IM, Culhane JF, McCollum KF, Matthew L, Elo IT. Literacy and depressive symptomatology among pregnant Latinas with limited English proficiency. Am J Orthopsychiatr. 2007;77:243248

43. Hahn EA, Cella D, Dobrez DG, et al. The impact of literacy on healthrelated quality of life measurement and outcomes in cancer outpatients. Qual Life Res. 2007;16:495-507.

44. Vinson J, Rich M, Sperry J, et al. Early readmission of elderly patients with congestive heart failure. J Am Geriat Soc. 1990;38:1290-1295.

45. Rogers A, Addington-Hall J, Abery A, et al. Knowledge and communication difficulties for patients with chronic heart failure: Qualitative study. Brit Med J. 2000;321:605-607.

46. Paasche-Orlow MK. Wolf MS The causal pathways linking health literacy to health outcomes. Am J Health Behav. 2007;31(Suppl1):S19-26. 